\title{
Design and Experimental Research of a New Kind of NC Rotary Swing Workbench
}

\author{
Lei Xing, Xiangdong Liu, Wei Lu, Yongcheng Jiang* and Fujun Ren \\ College of Mechanical Engineering, Jiamusi University, 154007, Jiamusi, China \\ *Corresponding author
}

\begin{abstract}
To realize the application of reciprocating electrical sparkle wire cutting technology and to solve the technical problems of sculptured surface machining, one new kind of NC rotary swing workbench has been studied based on computer aided technology. The advantages can be concluded as high precision and outstanding machining surface quality. The prototype of NC rotary swing workbench has been researched and some samples have been tested on it with good processing effect. On the basis of the summary and conclusion of all research works, some suggestions and prospects for further researches are put forwards.
\end{abstract}

Keywords-wire electronic discharge machining; spatial curved surface; five-axis linkage; programmable multi-axis controller

\section{INTRODUCTION}

Nowadays, so many moulds in machining electronic device, instrument, motor, airplane and missile have blanking taper or different shapes between up and down section view. So that the spatial curved surface parts processing technique in wire electronic discharge machining with high speed is one of the most important processing method. The wire tool can incline some degree by a special taper device to get spatial curved surface parts, but this processing method can generate error easily and the low automation degree of its NC system also reduce the processing quality of spatial curved surface parts. Therefore, the research on the key technique of intelligent NC system in wire electronic discharge machining with high speed is carried, A NC Rotary Table with Two Sways (RTTS) is proposed and researched deeply in this paper[1, 2].

Figure 1 is a schematic diagram of RTTS. The system comprises a mechanical drive system (including driving motors) and three implementing agencies. In the process of the spatial curved surface processing, five-axis linkages of $x, y, A$, $\mathrm{B}, \mathrm{C}$ axis are demanded, these motions can be operated by the numerical control swing workbench and the original wire cutting machine together. Currently there is no NC rotary swing workbench products which can realize special spatial curved surface cutting in our country. The design of NC rotary swing workbench is based on the motion analysis of the multi- axis machining system, the mathematical model of spatial curved wire cutting processing and the spatial model, which is the hardware basis for preparing a three-dimensional process control software.

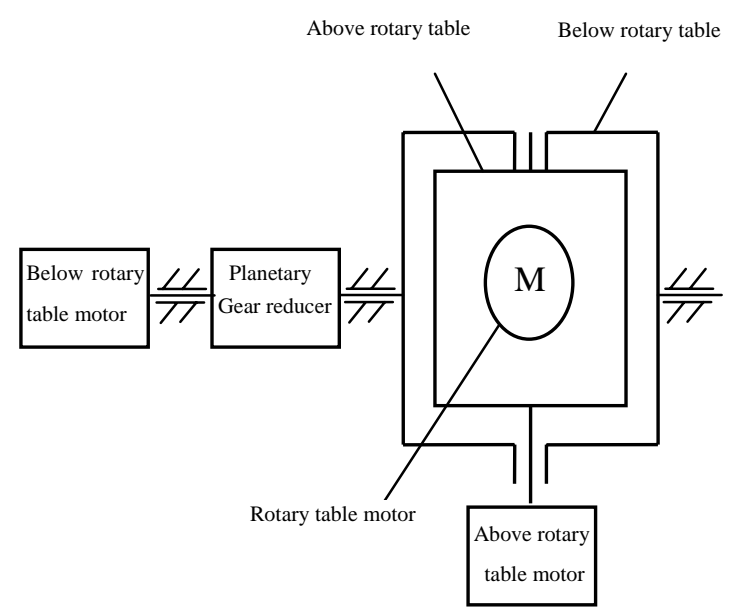

FIGURE I. SCHEMATIC DIAGRAM OF RTTS

\section{Modeling OF Rotary SWING WORKBEnCH UNDER PRO/E ENVIRONMENT}

A NC rotary swing workbench of five-axis linkages is built on the original wire electronic discharge machining reciprocal system by an additional three-axis CNC rotary swing workbench. Among them, the design of the "rotary" refers to the z-axis rotation around the coordinate space; two "swing" means swing respectively around the $\mathrm{x}$-axis, $\mathrm{y}$-axis swing achieved by three-axis linkages. Combined with the original wire electronic discharge machining reciprocal system, five-axis linkages can be achieved[3-5].

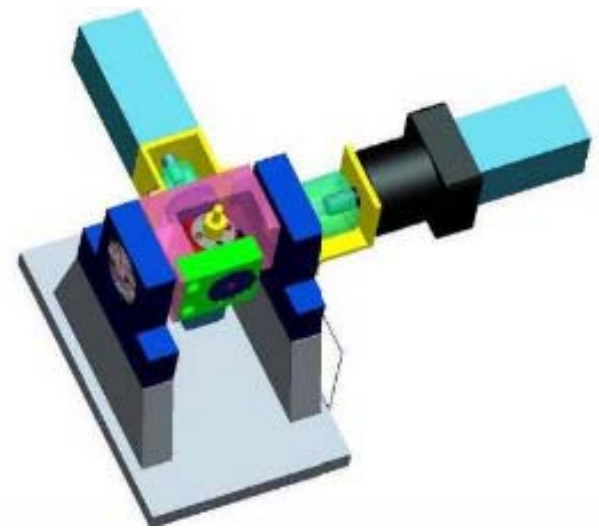

FIGURE II. 3D SOLID ASSEMBLED MODEL OF RTTS

During the spatial curved surface processing, the workpiece is generally fixed on the turntable, the axial is $\mathrm{C}$-axis which turning around the $\mathrm{z}$-axis, the turntable 
accumulates to rotate $360^{\circ}$, to achieve sub-degree rotation. A variety of space curved surface parts with the $\mathrm{x}, \mathrm{y}$-axis translation and the two rotations around $\mathrm{x}, \mathrm{y}$ can be processed [6-9]. Figure 2 shows a three-dimensional solid assembly model of RTTS under Pro/E software.

According to the motion characteristics analysis, summarizing and improving the workbench design, the test prototype of RTTS of five-axis linkages electronic discharge wire cutting has been designed and manufactured. The test prototype can form a complete set with the domestic standard, two-dimensional wire electronic discharge machining can realize the five-axis electronic discharge wire cutting or as a three-axis linkages device.

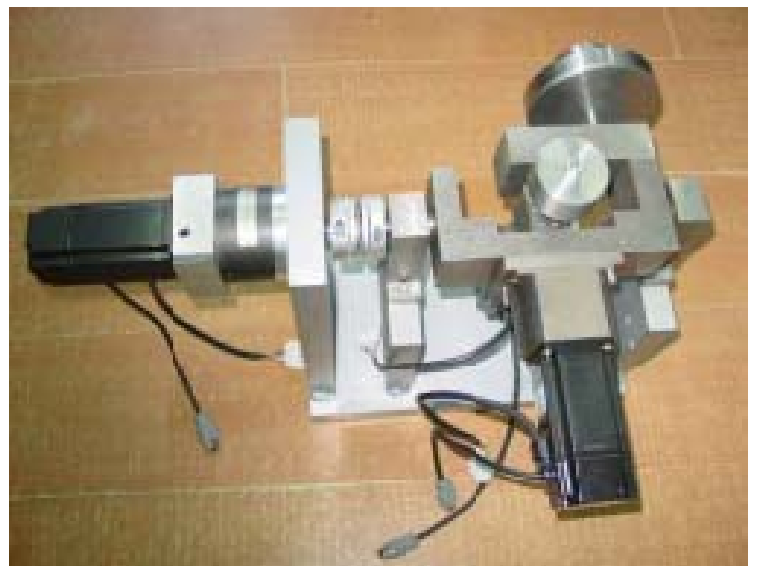

FIGURE III. TEST PROTOTYPE OF NC RTTS

Figure 3 shows the experimental prototype. The RTTS comprises the base, the below rotary table, planetary gear reducer, AC servo motor, the above rotary table, turntable, counterweight, flanges, fittings fixtures and other components.

The planetary gear reducer adopt Neugart PLE 40 gearbox made by Tianjin Luosheng drive Limited production, this planetary gear reducer is very wide used, with the advantages as low gap, high torque, high transmission efficiency (96\%), low noise, any installation position lifetime lubrication, etc., 22 kinds of reduction ratio can be available[10]. The output shaft of AC servo motor connects to the gear reducer input shaft through the coupling, an output angle of the motor after a mechanical breakdown can achieve higher indexing accuracy, and the output torque is increased 8 times, greatly enhanced the dynamic characteristics of RRTS.

The servo system used is the production of Panasonic AC servo system, driver model is MSMA042A1A, motor power is 400W. Servo motor drive mode uses the position control mode, and connects to an output terminal of PMAC Accessories ACC-8S. Servo motors adopts 2500 (pulse/rev) incremental encoder. Since the reduction gear ratio is $1: 8$, so the molecule of multiplication frequency of driver instruction pulse is set as 20000 , the equivalent angle of the turntable is ensured as $0.001^{\circ}$ corresponding to each pulse.

The above rotary table and the turntable directly connect to the servo motor via coupling, so the molecule of multiplication frequency of driver instruction pulse is set as 2
500 , the equivalent angle of the turntable is ensured as $0.001^{\circ}$ corresponding to each pulse. Figure 4 shows the test prototype of RTTS installs in the wire electronic discharge machining.

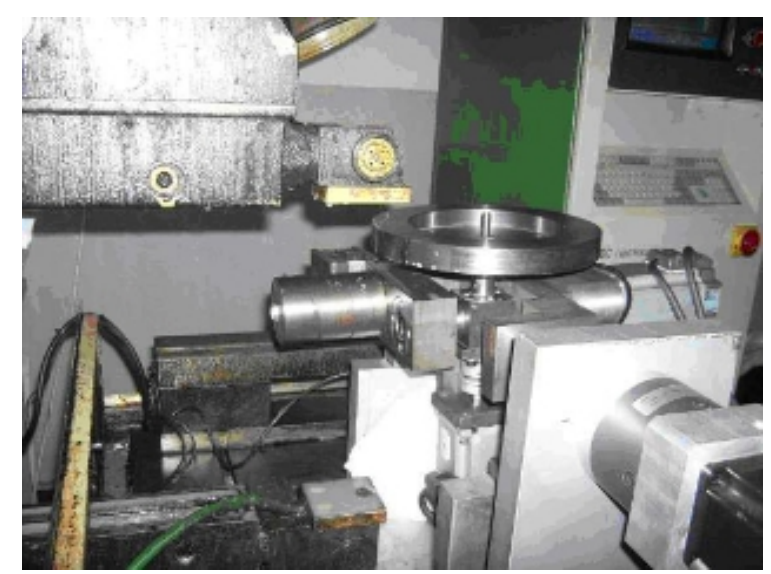

FIGURE IV. TEST PROTOTYPE OF NC RTTS WITH WIRE ELECTRONIC DISCHARGE MACHINING

\section{THE EXPERIMENT RESEARCH}

Test machining parameters are shown in Table 1. After the installation and adjustment of the blank, the relative position between the wire and the blank can be determined by the positioning mobile function and location-aware positioning function, then moving to the starting position coordinates of NC code, and setting this position as the programmed coordinates. Then entering the program processing function and running the NC code into the processing status, the main objective can be set as improving the processing efficiency, and also taking into account the discharge of the erosion product.

TABLE I. MACHINING PARAMETERS

\begin{tabular}{|c|c|c|c|}
\hline \multicolumn{2}{|l|}{ Pulse width $t_{i} / \mu s$} & \multicolumn{2}{|l|}{8} \\
\hline \multicolumn{2}{|c|}{ Pulse interval $t_{0} / \mu \mathrm{s}$} & \multicolumn{2}{|c|}{48} \\
\hline \multicolumn{2}{|l|}{ Peak current $I_{s} / A$} & \multicolumn{2}{|c|}{12} \\
\hline \multicolumn{2}{|c|}{ Thickness of the workpiece $h / \mathrm{mm}$} & \multicolumn{2}{|c|}{300} \\
\hline \multicolumn{2}{|c|}{ Cutting depth $a_{p} / \mu m$} & \multicolumn{2}{|c|}{10} \\
\hline \multirow{4}{*}{ Wire electrode } & Material & \multicolumn{2}{|c|}{ molybdenum } \\
\hline & Diameter $d / \mathrm{mm}$ & \multicolumn{2}{|c|}{0.18} \\
\hline & Wire speed $v_{s} / m \cdot s^{-1}$ & 5 & 10.3 \\
\hline & Tension $T_{w} / N$ & \multicolumn{2}{|c|}{8.0} \\
\hline
\end{tabular}

Some the spatial curved surface samples can be got by the actual tests. Figure 5 is the machining process diagram of space curved parts. Figure 6 is the spatial curved surface sample which the top lead is Archimedes spiral and bottom lead is circle curves. Figure 7 shows the spatial curved surface samples which the top lead and bottom lead are all circle curves. Figure 8 is the spatial curved surface sample which the top lead is diamond and the bottom lead is composed with line and circle curves. 


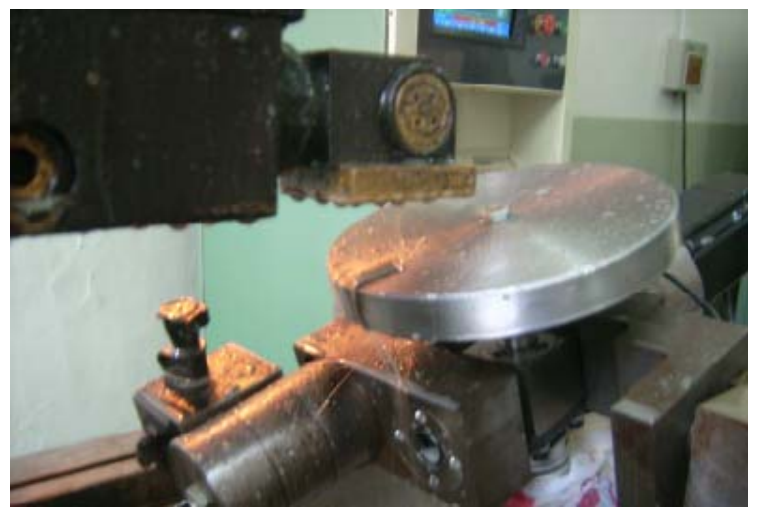

FIGURE V. MACHINING PROCESS OF THE SPATIAL CURVED SURFACE PARTS

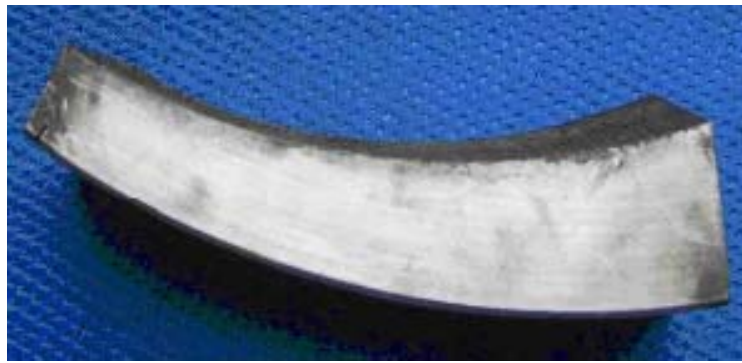

FIGURE VI. SAMPLE WITH THE TOP LEAD ARCHIMEDES SPIRAL AND THE BOTTOM LEAD CIRCLE CURVES

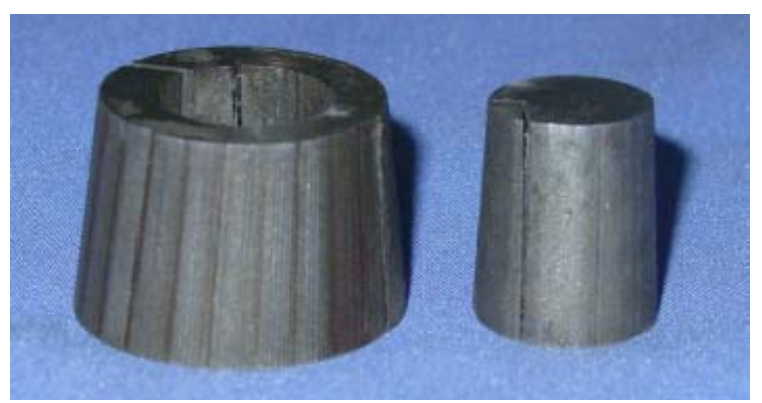

FIGURE VII. SAMPLE WITH THE TOP LEAD AND THE BOTTOM LEAD ALL CIRCLE CURVES

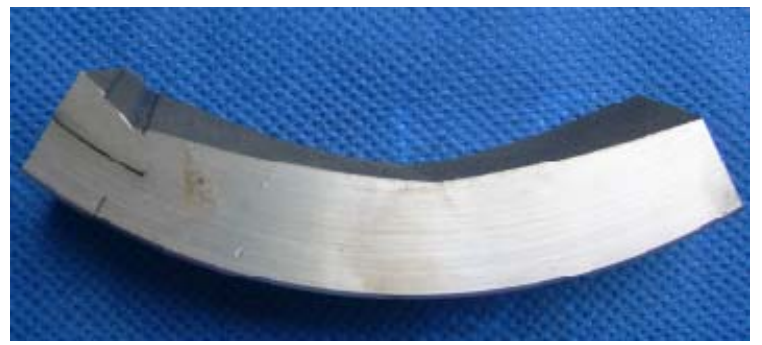

FIGURE VIII. SAMPLE WHICH THE TOP LEAD DIAMOND AND THE BOTTOM LEAD COMPOSED WITH LINE AND CIRCLE CURVES

\section{PROCESSING ERROR ANALYSIS}

As shown in Figure 9, in the actual processing, the swing turntable of RTTS must always swing back determined by the spatial curved surface characteristics, and $\mathrm{x}, \mathrm{y}$ axis motion must often change in order to achieve the spatial curved surface processing purpose. Due to the mechanical structure of the workbench and the lead screw table, the workpiece will produce a certain gap in the process of swing back and reciprocating motion, causing a certain error during the workpiece swing back, so that the actual swing angle obtained is smaller than expected, resulting in the overall size of parts should be larger than the simulation results. To improve the processing status, not only the manufacturing precision of the workbench must improve, but also the high-precision lead screw must be selected.

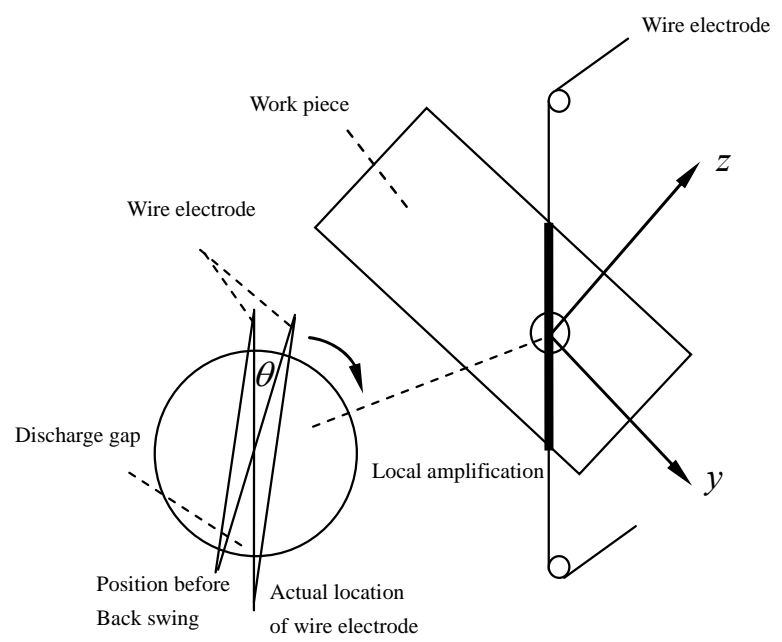

FIGURE IX. SWAY BACK ERROR OF RTTS

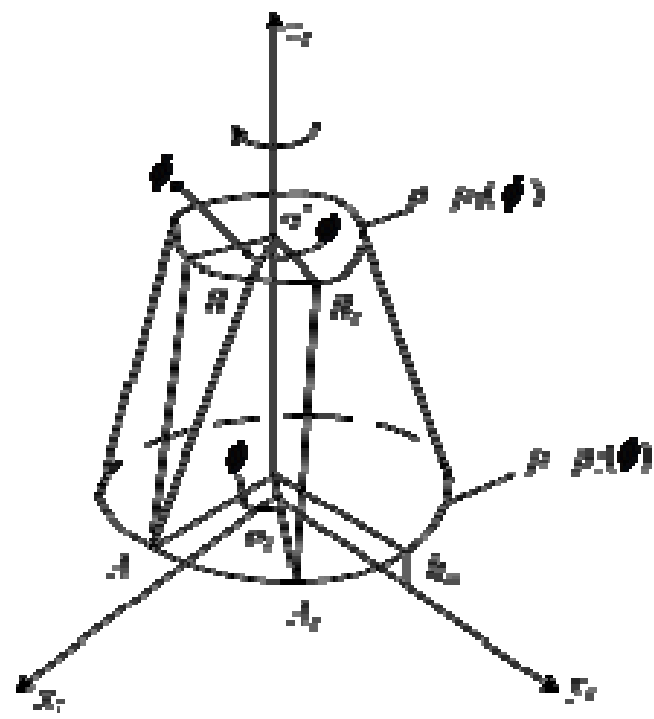

FIGURE X. MOTION COORDINATE SYSTEM OF FIVE-AXIS LINKAGE MACHINING SYSTEM

Figure 10 shows the workpiece rotation $\phi=\omega t$ through the elapsed time t, the upper conductor $\rho=\rho_{1}(\phi)$, the below conductor $\rho=\rho_{2}(\phi)$ can obtain a polar radius value, respectively. Through the variation of polar radius values, the 
motion amount of $\mathrm{x}, \mathrm{y}, \mathrm{A}, \mathrm{B}, \mathrm{C}$ axis can be obtained, so that the other spatial location of conductor can be determined at time t. Connecting the two locations with a line, this line is the actual workpiece walking paths. Thus, the CNC model determines the wire motion fitting a straight line but not as a vertical wire walking parametric equation, resulting in a processing principle error. The tiny ripples produce in the actual processing, especially the upper and the below conductor composes by several parametric equation paragraphs, the shape distortion of the workpiece is likely to produce at the intersections of the two.

Further, since the workpiece constantly swings in the processing period, the size of the wire electrode and the workpiece surface always changes. Thus, the spatial curved surface parts are the varying-size process, and the size change will inevitably lead to the process state change, it will also cause the workpiece errors.

\section{CONCLUSIONS}

The processing test of spatial curved surface parts have been carried, the results obtained by processing and simulation are basically similar, the multi-axis RTTS system has been verified good possessing stability, fast real-time response ability and strong expansibility. The correctness of mathematical models and the rationality design of RTTS has also been verified. The main reasons influencing the machining accuracy are analyzed as the following: the swing back of the workbench and the backlash of the lead screw reducing machining accuracy; electrode wire deformation caused by the workpiece swing also affect the machining accuracy; the varying-size process caused by the cutting table swing has a negative impact on the processing error.

\section{ACKNOWLEDGEMENT}

This research was financially supported by the Natural Science Foundation of Heilongjiang province (E9815).

\section{REFERENCES}

[1] He Jing, Wang Gang, De-wei Wu. Military navigation system virtual prototyping supporting platform to study. J. Jurnal of system simulation. 2008, (20) pp. 550-552.

[2] N Özdemir, Cebeli Özek. An Investigation on Mach inability of Nodular Cast Iron by WEDM.J. The International Journal of Advanced Manufacturing Technology. 2006, (28) pp. 9.

[3] Ran Chong Du Jing, Yu-xia Liu. Motorcycle ride comfort simulation analysis based on virtual prototype technology and suspension optimization design.J. Modern manufacturing engineering. 2008, (1) pp. 21-23.

[4] Yu Lin, Guang-chen Bai, Jun-ting Jiao, etc. The virtual prototype of space station deploy able structure simulation and reliability analysis .J. Journal of system simulation. 2007, (19) pp. 78-80.

[5] Aminollah Mohammadi, Alireza Fadaei Tehrani, Ehsan Emanian, et al. A new Approach to Surface Roughness and Roundness Improvement in Wire Electrical Discharge Turning based on Statistical Analyses[J]. The International Journal of Advanced Manufacturing Technology, 2007, (19) 7-17.

[6] R Ramakrishnan, L Karunamoorthy. Multi Response Optimization of Wire EDM Operations Using Robust

[7] Design of Experiments[J]. The International Journal of Advanced Manufacturing Technology, 2006, (29) pp. 1-2.
[8] Sang Min Yi, Min Soo Park, Young Soo Lee, et al. Fabrication of a Stainless Steel Shadow Mask using Batch Mode Micro-EDM[J]. Microsystem Technologies, 2008, (14) pp. 3.

[9] H Ramasawmy, L Blunt. Investigation of the Effect of Electrochemical Polishing on EDM Surfaces[J]. The International Journal of Advanced Manufacturing Technology, 2007, (31) pp. 11-12.

[10] J S Kuo, K T Chiu, S W Hsu, et al. A Novel Technique for the Fabrication of Herringbone Grooves in a Dynamic Thrust Bearing Combining UV-L[J]

[11] Microsystem Technologies, 2006, (12) pp. 6. 